\title{
La industria de la construcción privada: su participación en el PBI de Moquegua, 2013-2017
}

\author{
The private construction industry: its participation in Moquegua's PBI, 2013-2017
}

Brady Manuel Vilcanqui Chambi

Unidad de Logística. Administración de Empresas SAC. Lima - Perú.

E - mail: bvilcanqui@gmail.com

\section{RESUMEN}

La investigación tuvo como objetivo determinar la incidencia de la inversión privada del sector construcción, tanto de la inversión de capital, las licencias de construcción tramitadas y la mano de obra calificada, en el producto bruto interno de Moquegua, desde el 2013 al 2017. El método de la investigación es de tipo básico, explicativo, con diseño no experimental y con método retrospectivo, aplicando la técnica de análisis documental, recurriendo a información registrada en las instituciones públicas, cuyos resultados se analizaron estadísticamente a través de la prueba regresión lineal. Se concluye que la inversión privada del sector construcción ha incidido directamente en el producto bruto interno de Moquegua, desde el 2013 al 2017, con una tendencia en crecimiento, positivo y constante.

Palabras clave: Inversión privada, producto bruto interno, sector construcción.

\begin{abstract}
This research aimed to determine the incidence of private investments in the construction sector as the capital investment, the construction licenses processed and skilled labor in Moquegua's Gross Domestic Product from 2013 to 2017. The method used this research is basic and explanatory non experimental with a retrospective study design applying document analysis and using available information from public institutions, whose results were statistically analyzed through linear regression. It is concluded that private investment in the construction sector has directly incidence in Moquegua's Gross Domestic Product from 2013 to 2017, with a growing, positive, and constant tendency.
\end{abstract}

Keywords: Private investment, construction sector, gross domestic product.

\section{INTRODUCCIÓN}

El Perú fue el décimo primer país de América Latina con la mayor tasa de inversión privada y pública en el 2017, llegando a representar alrededor del $22 \%$ de su Producto Bruto Interno (PBI), según el informe La Hora de la Transformación del Banco Interamericano de Desarrollo (BID); sin embargo, aún se sitúa por debajo de la tasa de inversión promedio de Europa emergente, que llega a alrededor del $25 \%$ del PBI.

El ranking del Índice de Competitividad Regional del Instituto Peruano de Economía (Lezama, 2018) colocaba a Moquegua en el tercer puesto en el 2012; sin embargo, alcanzó el segundo lugar en el 2013 hasta el 2017. Esta situación se debe gracias a su actividad productiva que se centra principalmente en los sectores minería, manufactura, construcción y servicios.

Según el Banco Central de Reserva del Perú (2016), entre el 2008 y 2015, Moquegua registró un crecimiento promedio anual de 1,9\% del Valor Agregado Bruto (VAB), inferior al registrado a nivel nacional (5.2\%). Entre 
las actividades que más se destacan en su aporte a la economía de Moquegua, se tiene la manufactura, ligada a la minera, con el $44.3 \%$; seguido de la minería con el $32.5 \%$; otros servicios con el 6.0 \% y construcción con el 5.9 $\%$. El sector construcción que, incluye además de la actividad inmobiliaria la construcción de infraestructura, registró durante el 2015 un crecimiento negativo de $-5.8 \%$.

En Moquegua en el periodo enero-agosto del 2018, el sector construcción creció en 5.04 \%, como resultado de la evolución positiva del avance físico de obras en $8.65 \%$ y del consumo interno de cemento en $4.19 \%$. En agosto 2018, el Índice de la Producción del sector Construcción registró una disminución de -0.09 \% por la baja registrada en el avance físico de obras, según el informe técnico del Instituto Nacional de Estadística e Informática [INEI]; sin embargo, se registró un incremento del consumo interno de cemento, debido al aumento de la actividad constructora de obras privadas del sector minero, edificaciones residenciales, centros comerciales, centros educativos y casas rurales (Flores, 2018).

La revisión de los antecedentes se presenta en línea siguientes, Mero, Herrera y Herrera (2018), en su tesis titulada: "Influencia de la sostenibilidad en el sector de la construcción en Ecuador sobre el producto interno bruto PIB periodo 2010-2016"; presentada en la Universidad de Guayaquil en Ecuador, desarrollaron una investigación documental, recurriendo a los boletines de series de información de cuentas nacionales producción de las industrias - miles de dólares 2007-2018. Concluyeron que existe influencia significativa de la sostenibilidad en el sector construcción, el cual ha tenido una participación del $10 \%$ anual, sobre el Producto Interno Bruto (PIB).

Pineda (2013), en su tesis titulada: "Análisis de la productividad y sus determinantes en el sector construcción del Ecuador en base al Censo Económico", presentada en la Facultad Latinoamericana de Ciencias Sociales en Ecuador, realiza un estudio descriptivo, no experimental y retrospectivo, recurriendo a datos del Censo Económico del país, concluyendo que factores, como investigación y desarrollo, y capacitación a los empleados, no resultaron ser determinantes para la productividad de la empresa, los cuales no afectan directamente en el crecimiento económico de la región.

Frey y Vela (2014), en su tesis titulada: "Relevancia del proceso de abastecimiento en empresas grandes del sector construcción: Estudio de caso comparado entre empresas familiares peruanas"; presentada en la Pontificia Universidad Católica del Perú, realiza un estudio descriptivo y no experimental, utilizando como técnica la entrevista semi-estructurada dirigido a ICCGSA y COSAPI. Concluye que el sector construcción es un actor relevante en el crecimiento socioeconómico y el desarrollo del país, principalmente porque su actividad incide en la reducción de la brecha de infraestructura del país que es una de las más altas de la región, así como contribuye a reducir el déficit de viviendas; ambas situaciones hacen al sector construcción peruano atractivo para la inversión pública y privada.

Vargas, Castro y Bautista (2011), en su investigación titulada: "Importancia del crecimiento del sector construcción en la economía y sociedad peruana", presentada en la Universidad Nacional Mayor de San Marcos, realiza un estudio exploratorio, analizando datos a través de entrevistas a profundidad a expertos de la industria de construcción y de fuentes secundarias como Apoyo y Maximixe. Concluyen que el sector construcción continuará siendo uno de los principales motores del crecimiento económico del país, aproximadamente, el $8 \%$ anual sostenido para los próximos años.

Jiménez (2010) plantea que mientras la inversión incrementa la capacidad productiva, su efecto sobre los beneficios y el producto es poco significativo. Por otro lado, cuando el gobierno estimula la economía, el déficit comercial se incrementa junto con el déficit público. Por ello, el auge produce crisis de balanza de pagos, seguida por una deuda pública externa creciente; asimismo, el gasto público que no crea capacidad, compensa la pérdida de demanda efectiva y, por tanto, previene la disminución de los índices de beneficios. El Estado no puede reducir simultáneamente los déficits público y externo sin afectar los beneficios, el producto y el empleo.

Kogan, y Bondorevsky (2016) mencionan que la inversión en América Latina está en el orden del $3 \%$ del PIB por año, por debajo del nivel aconsejado en diversos estudios, que es del $5 \%$. Para lograr esos niveles de inversión en Latinoamérica es necesaria la inversión proveniente del sector público y privado, la cual se estima que, actualmente, el $30 \%$ es privado. 
Mendoza (2016) menciona que la teoría económica sugiere que la inversión privada depende de los términos de intercambio y de la velocidad de expansión de la actividad económica. Por otro lado, un crecimiento más rápido del producto aumenta la inversión pues las empresas anticipan un mayor tamaño de mercado.

De igual manera, Jiménez (2010) menciona que la inversión crea capacidad, pero añade muy poco a la demanda de bienes producidos en el país precisamente porque sus efectos multiplicadores son exportados. Por esta razón, la demanda interna pasa a depender fundamentalmente del déficit gubernamental, el cual crea demanda efectiva sin aumentar directamente la capacidad productiva industrial.

Por otro lado, Campos y Carcelén (2003) definen el sector construcción como "uno de los sectores más dinámicos de la economía, pues sus actividades involucran a otras industrias relacionadas, es así, que muchas veces se asocia el crecimiento del sector con el desarrollo de la economía de un país" (p.1).

Umaña (2003) sostiene que el sector de la construcción afecta la economía en tres niveles: familia, empresa y Estado. Demanda tierra, capital, mano de obra, maquinaria, materiales, bienes, servicios, tecnología, servicios públicos, telecomunicaciones y recursos financieros. Realiza obras civiles y de edificación, que generan salarios, ingresos, utilidades, intereses e impuestos.

La inversión se basa en un comportamiento optimizador de las firmas, sujeto a la trayectoria de las tasas de interés, los precios del capital y las políticas tributarias (Cerda, y Larraín, 2005).

La inversión de capital es un término utilizado para designar al dinero que una empresa invierte en activos físicos, mercaderías y materiales de producción para operar en el mercado, cuya rentabilidad se percibe a largo plazo si las decisiones tomadas en qué invertir son acertadas y eficientes. Estos activos pueden ser maquinarias, vehículos de carga y especiales para la construcción, equipos de cómputo, vehículos de transporte, herramientas digitales de medición, entre otros.

Mero, Herrera, y Herrera (2018) precisan que "la formación de un desarrollo dinámico estable del sector de la construcción solo es posible sobre la base de una gestión eficaz de las actividades de inversión donde existe una alta probabilidad de efecto sinérgico positivo" (p.2).

La empresa puede financiarse a través de recursos propios, recursos ajenos o mediante una combinación de ambas fuentes, sin embargo, necesitará conocer, por un lado, el coste de cada fuente de financiación que tiene la posibilidad de utilizar y que le permitirá llevar a cabo las inversiones que ha previsto y, por el otro, el coste de capital medio ponderado del conjunto de fuentes utilizadas (Verona y Hernández, 2010).

La sociedad demanda edificios habitacionales como departamentos y viviendas; sin embargo, estas infraestructuras generan problemas ambientales durante su construcción, operación y mantenimiento, y destrucción, que en algunos casos contravienen las normas ambientales. (Mero, Herrera y Herrera, 2018)

Vyas, Ahmed, y Parashar (2014) mencionan que, en el caso de los edificios que consumen gran cantidad de energía y recursos naturales no renovables, estos impactan en el cambio climático al afectar la calidad del aire, el agua y el suelo en las ciudades.

El objetivo de la investigación es determinar la incidencia de la inversión privada del sector construcción en el producto bruto interno de Moquegua, desde el 2013 al 2017.

Y por otro lado se ha formulado la siguiente pregunta para la investigación en cuestión:

¿Cómo ha incidido la inversión privada del sector construcción en el producto bruto interno de Moquegua, en el 2013 al 2017?

\section{Hipótesis de investigación:}

La inversión privada del sector construcción ha incidido significativamente en el producto bruto interno de Moquegua, desde el 2013 al 2017.

Las hipótesis específicas son:

Hipótesis específica 1: La inversión de capital del sector construcción ha presentado un impacto importante en 
el producto bruto interno de Moquegua, desde el 2013 al 2017.

Hipótesis específica 2: La gestión de licencias del sector construcción ha influido significativamente en el producto bruto interno de Moquegua, desde el 2013 al 2017.

Hipótesis específica 3: La consecución de mano de obra calificada del sector construcción ha incidido notablemente en el producto bruto interno de Moquegua, desde el 2013 al 2017.

\section{MÉTODOS Y MATERIALES}

La población que abarca la investigación está conformada por información registrada sobre inversión de capital, licencias de construcción tramitadas, mano de obra calificada e índices del producto bruto interno que proporcionan la Dirección Regional de la Producción, Municipalidad Provincial Mariscal Nieto, SENCICO y Ministerio de Economía y Finanzas, respectivamente; en el periodo 2013-2017.

Como variables que explican la variable dependiente (PBI) se consideran:

Inversión privada en el sector de construcción, capital invertido, número de licencias de construcción otorgadas por las municipalidades, contratación de mano de obra calificada.

\section{RESULTADOS}

A continuación, se presentan los resultados de cada una de las relaciones de las variables que intervienen en la investigación.

Tabla 1. Inversión de capital en el sector construcción, Moquegua 2013 - 2017

\begin{tabular}{lrrrrr}
\hline $\begin{array}{l}\text { Elemento de } \\
\text { medición }\end{array}$ & $\mathbf{2 0 1 3}$ & $\mathbf{2 0 1 4}$ & $\mathbf{2 0 1 5}$ & $\mathbf{2 0 1 6}$ & $\mathbf{2 0 1 7}$ \\
\hline $\begin{array}{l}\text { Consumo interno } \\
\text { de cemento (Tn) }\end{array}$ & 11135679 & 11441392 & 11186304 & 10909271 & 10925485 \\
$\begin{array}{l}\text { Venta de barras } \\
\text { de construcción } \\
(\text { Tn })\end{array}$ & 1191064 & 1338472 & 1316263 & 1304285 & 1309008 \\
$\begin{array}{l}\text { Venta interna de } \\
\text { asfalto (barril) }\end{array}$ & 1348191 & 1669098 & 1409983 & 1322891 & 1653293 \\
\hline
\end{tabular}

Fuente: Series Nacionales (INEI, 2019).

De acuerdo a la tabla 1, se observa que el consumo interno de cemento alcanzó el nivel más alto en el 2014 (con 11 441392 toneladas) y el más bajo en el 2016 (con 10925485 toneladas), con una variación de $-1.9 \%$ entre 2013 y 2017; respecto a la venta de barras de construcción, se alcanzó el nivel más alto en el 2014 (con 1338472 toneladas) y el más bajo en el 2013 (con 1191064 toneladas), con una variación de $+9.9 \%$ entre 2013 y 2017; y, respecto a la venta interna de asfalto, se alcanzó el nivel más alto en el 2014 (con 1669098 barriles) y el más bajo en el 2016 (con 1322891 barriles), con una variación de $+22.6 \%$ entre 2013 y 2017.

Tabla 2. Licencias de construcción tramitadas en el sector construcción, Moquegua 2013 - 2017

\begin{tabular}{cccc}
\hline & \multicolumn{3}{c}{ Total } \\
\cline { 2 - 4 } Año & Número & Variación del año anterior \\
\hline 2013 & 82 & - & - \\
2014 & 92 & +10 & $+12.2 \%$ \\
2015 & 105 & +13 & $+14.1 \%$ \\
2016 & 119 & +14 & $+13.3 \%$ \\
2017 & 135 & +16 & $+13.4 \%$
\end{tabular}

Fuente: Municipalidad Provincial de Mariscal Nieto - Moquegua (2019). 
Según la tabla 2, se observa un incremento porcentual constante de licencias de construcción tramitadas por la Municipalidad Provincial Mariscal Nieto desde el 2013 hasta el 2017, con una variación de $+64.6 \%$, lo cual representa un crecimiento importante en la cantidad de unidades en infraestructura en construcción dentro de la provincia Mariscal Nieto.

Tabla 3.1. Mano de obra calificada en el sector construcción, Moquegua 2013 - 2017

\begin{tabular}{cccc}
\hline Año & \multicolumn{3}{c}{ Total } \\
\cline { 2 - 4 } & Número & Variación del año anterior \\
\hline 2013 & 135 & - & - \\
2014 & 142 & 7 & $+5.2 \%$ \\
2015 & 145 & 3 & $+2.1 \%$ \\
2016 & 151 & 6 & $+4.1 \%$ \\
2017 & 163 & 12 & $+7.9 \%$ \\
\hline
\end{tabular}

Fuente: Servicio Nacional de Capacitación para la Industria de la Construcción (2019).

Según la tabla 3.1, se observa un incremento porcentual regular de mano de obra calificada por el Servicio Nacional de Capacitación para la Industria de la Construcción - SENCICO, desde el 2013 hasta el 2017, con una variación de $+20.7 \%$, lo cual representa un crecimiento notable en la cantidad de mano de obra calificada que está a disposición del sector construcción dentro de la región Moquegua.

Tabla 3.2. Producto bruto interno del sector construcción, Moquegua 2013 - 2017

\begin{tabular}{cccc}
\hline \multirow{2}{*}{ Año } & \multicolumn{3}{c}{ Total } \\
\cline { 2 - 4 } & PBI (miles de soles) & Variación del año anterior \\
\hline 2013 & 8598669 & - & - \\
2014 & 8670803 & 72134 & $+0.8 \%$ \\
2016 & 8905344 & 234541 & $+2.7 \%$ \\
2017 & 9139875 & 234531 & $+2.6 \%$ \\
\hline
\end{tabular}

Fuente: Instituto Nacional de Estadística e Informática (2019).

Y luego, a partir de los resultados obtenidos, se contrasta las hipótesis de la investigación:

Formulación de la hipótesis general:

$\mathrm{H}_{0}$ : $\quad$ La inversión privada del sector construcción no ha incidido significativamente en el producto bruto interno de Moquegua, desde el 2013 al 2017.

$\mathrm{H}_{\mathrm{i}}$ : La inversión privada del sector construcción ha incidido significativamente en el producto bruto interno de Moquegua, desde el 2013 al 2017.

Nivel de significancia

$\propto=0,05$

Pruebas de normalidad 
Tabla 4. Pruebas de normalidad para el contraste de la hipótesis general

\begin{tabular}{lcccrrr}
\hline & \multicolumn{2}{c}{ Kolmogorov-Smirnov } & \multicolumn{3}{c}{ Shapiro-Wilk } \\
& Estadístico & gl & Sig. & Estadístico & gl & Sig. \\
\hline Inversión & 0.154 & 20 & 0.020 & 0.891 & 20 & 0.029 \\
PBI & 0.197 & 20 & 0.040 & 0.818 & 20 & 0.002 \\
\hline
\end{tabular}

Fuente: datos procesamiento en Eviews.

Se observa que el valor Sig. (p-valor), tanto para Kolmogorov-Smirnov y Shapiro-Wilk, son menores a 0.05 (grado de significancia), por tanto, se puede afirmar que los datos no provienen de una distribución normal, el cual es requerimiento para aplicar la prueba de regresión lineal.

Tabla 5. Regresión lineal para el contraste de la hipótesis general

\begin{tabular}{ccrrr}
\hline Modelo & R & R cuadrado & $\begin{array}{r}\text { R cuadrado } \\
\text { corregida }\end{array}$ & $\begin{array}{r}\text { Error típico de la } \\
\text { estimación }\end{array}$ \\
\hline 1 & 0.981 & 0.962 & 0.960 & 83196.061 \\
\hline
\end{tabular}

Fuente: datos procesamiento en Eviews.

Tabla 6. ANOVA para el contraste de la hipótesis general

\begin{tabular}{crrrrr}
\hline Modelo & Suma de cuadrados & gl & Media cuadrática & F & Sig. \\
\hline Regresión & $3.170 \mathrm{E}+12$ & 1 & $3.170 \mathrm{E}+12$ & 458.058 & 0.000 \\
Residual & $1.246 \mathrm{E}+11$ & 18 & 6921584507 & & \\
Total & $3.295 \mathrm{E}+12$ & 19 & & & \\
\hline
\end{tabular}

Fuente: datos procesamiento en Eviews.

Tabla 7. Coeficientes para el contraste de la hipótesis general

\begin{tabular}{crrrrr}
\hline \multirow{2}{*}{ Modelo } & \multicolumn{2}{c}{ Coeficientes no estandarizados } & $\begin{array}{c}\text { Coeficientes } \\
\text { estandarizados }\end{array}$ & t & Beta \\
\cline { 2 - 5 } & Beta & Error típico & & 1.198 & 0.241 \\
\hline (Constante) & 11.688 & 9.755 & & & \\
Inversión & 0.860 & 0.135 & 0.769 & 6.369 & 0.000 \\
\hline
\end{tabular}

Fuente: datos procesamiento en Eviews.

Se observa un alto coeficiente de determinación $\left(\mathrm{r}^{2}: 0.962\right)$ y un alto índice de correlación (r: 0.981$)$; asimismo, la Sig. (p-valor) es menor a 0.05 (grado de significancia), por lo cual se rechaza la hipótesis nula y se afirma que existe influencia directa y significativa de una variable sobre la otra.

Contraste de la hipótesis específica 1

Formulación de la hipótesis nula $\left(H_{0}\right)$

$\mathrm{H}_{0}$ : $\quad$ La inversión de capital del sector construcción no ha presentado un impacto importante en el producto bruto interno de Moquegua, desde el 2013 al 2017.

$\mathrm{H}_{\mathrm{i}}$ : $\quad$ La inversión de capital del sector construcción ha presentado un impacto importante en el producto bruto interno de Moquegua, desde el 2013 al 2017. 


\section{Nivel de significancia}

$$
\propto=0,05
$$

Pruebas de normalidad

Tabla 8. Pruebas de normalidad para el contraste de la hipótesis específica 1

\begin{tabular}{lccrrrr}
\hline & \multicolumn{2}{c}{ Kolmogorov-Smirnov } & \multicolumn{3}{c}{ Shapiro-Wilk } \\
& Estadístico & gl & Sig. & Estadístico & gl & Sig. \\
\hline Capital & 0.234 & 20 & 0.005 & 0.828 & 20 & 0.002 \\
PBI & 0.197 & 20 & 0.040 & 0.818 & 20 & 0.002 \\
\hline
\end{tabular}

Fuente: datos procesamiento en Eviews.

Se observa que el valor Sig. (p-valor), tanto para Kolmogorov-Smirnov y Shapiro-Wilk, son menores a 0.05 (grado de significancia), por tanto, se puede afirmar que los datos no provienen de una distribución normal, el cual es requerimiento para aplicar la prueba de regresión lineal.

Prueba de regresión linealy decisión

Tabla 9. Regresión lineal para el contraste de la hipótesis específica 1

\begin{tabular}{ccccr}
\hline Modelo & R & R cuadrado & $\begin{array}{r}\text { R cuadrado } \\
\text { corregida }\end{array}$ & $\begin{array}{r}\text { Error típico de la } \\
\text { estimación }\end{array}$ \\
\hline 1 & 0.730 & 0.533 & 0.507 & 292435.132 \\
\hline
\end{tabular}

Fuente: datos procesamiento en Eviews.

Tabla 10. ANOVA para el contraste de la hipótesis especifica 1

\begin{tabular}{crrrrr}
\hline Modelo & Suma de cuadrados & gl & Media cuadrática & F & Sig. \\
\hline Regresión & $1.756 \mathrm{E}+12$ & 1 & $1.756 \mathrm{E}+12$ & 20.531 & 0.000 \\
Residual & $1.539 \mathrm{E}+12$ & 18 & 85518306649 & & \\
Total & $3.295 \mathrm{E}+12$ & 19 & & & \\
\hline
\end{tabular}

Fuente: datos procesamiento en Eviews.

Tabla 11. Coeficientes para el contraste de la hipótesis especifica 1

\begin{tabular}{|c|c|c|c|c|c|}
\hline \multirow{2}{*}{ Modelo } & \multicolumn{2}{|c|}{ Coeficientes no estandarizados } & \multirow{2}{*}{$\begin{array}{r}\begin{array}{r}\text { Coeficientes } \\
\text { estandarizados }\end{array} \\
\text { Beta }\end{array}$} & \multirow{2}{*}{$\mathbf{t}$} & \multirow{2}{*}{ Sig. } \\
\hline & Beta & Error típico & & & \\
\hline (Constante) & 25901201.03 & 3728872.790 & & 6.946 & 0.000 \\
\hline Inversión & -1.519 & 0.335 & -0.730 & -4.531 & 0.000 \\
\hline
\end{tabular}

Fuente: datos procesamiento en Eviews.

Se observa un coeficiente regular de determinación $\left(\mathrm{r}^{2}: 0.533\right)$ y un índice medio de correlación (r: 0.730); asimismo, la Sig. (p-valor) es menor a 0.05 (grado de significancia), por lo cual se rechaza la hipótesis nula y se afirma que existe influencia directa y significativa de una dimensión sobre la otra.

Contraste de la hipótesis específica 2

Formulación de la hipótesis nula $\left(H_{0}\right)$ 
$\mathrm{H}_{0}$ : $\quad$ La gestión de licencias del sector construcción no ha influido significativamente en el producto bruto interno de Moquegua, desde el 2013 al 2017.

$\mathrm{H}_{\mathrm{i}}$ : $\quad$ La gestión de licencias del sector construcción ha influido significativamente en el producto bruto interno de Moquegua, desde el 2013 al 2017.

Nivel de significancia

$$
\propto=0.05
$$

Pruebas de normalidad

Tabla 12. Pruebas de normalidad para el contraste de la hipótesis especifica 2

\begin{tabular}{lrrrrrr}
\hline & \multicolumn{2}{c}{ Kolmogorov-Smirnov } & \multicolumn{3}{c}{ Shapiro-Wilk } \\
& Estadístico & gl & Sig. & Estadístico & gl & Sig. \\
\hline Licencias & 0.175 & 20 & 0.011 & 0.888 & 20 & 0.025 \\
PBI & 0.197 & 20 & 0.040 & 0.818 & 20 & 0.002 \\
\hline
\end{tabular}

Fuente: datos procesamiento en Eviews.

Se observa que el valor Sig. (p-valor), tanto para Kolmogorov-Smirnov y Shapiro-Wilk, son menores a 0.05 (grado de significancia), por tanto, se puede afirmar que los datos no provienen de una distribución normal, el cual es requerimiento para aplicar la prueba de regresión lineal.

\section{Prueba de regresión linealy decisión}

Tabla 13. Regresión lineal para el contraste de la hipótesis especifica 2

\begin{tabular}{crrrr}
\hline Modelo & R & R cuadrado & $\begin{array}{r}\text { R cuadrado } \\
\text { corregida }\end{array}$ & $\begin{array}{r}\text { Error típico de la } \\
\text { estimación }\end{array}$ \\
\hline 1 & 0,970 & 0,941 & 0,938 & 103629,356 \\
\hline
\end{tabular}

Fuente: datos procesamiento en Eviews.

Tabla 14. ANOVA para el contraste de la hipótesis específica 2

\begin{tabular}{crrrrr}
\hline Modelo & Suma de cuadrados & gl & Media cuadrática & F & Sig. \\
\hline Regresión & $3.102 \mathrm{E}+12$ & 1 & $3.102 \mathrm{E}+12$ & 288.831 & 0.000 \\
Residual & $1.933 \mathrm{E}+11$ & 18 & 10739043475 & & \\
Total & $3.295 \mathrm{E}+12$ & 19 & & & \\
\hline
\end{tabular}

Fuente: datos procesamiento en Eviews.

Tabla 1. Coeficientes para el contraste de la hipótesis especifica 2

\begin{tabular}{|c|c|c|c|c|c|}
\hline \multirow{2}{*}{ Modelo } & \multicolumn{2}{|c|}{ Coeficientes no estandarizados } & \multirow{2}{*}{$\begin{array}{r}\begin{array}{r}\text { Coeficientes } \\
\text { estandarizados }\end{array} \\
\text { Beta }\end{array}$} & \multirow{2}{*}{ t } & \multirow{2}{*}{ Sig. } \\
\hline & Beta & Error típico & & & \\
\hline (Constante) & 6783797.993 & 132909.664 & & 51.041 & 0.000 \\
\hline Inversión & 20865.004 & 1227.712 & 0.970 & 16.995 & 0.000 \\
\hline
\end{tabular}

Fuente: datos procesamiento en Eviews. 
Se observa un alto coeficiente de determinación $\left(\mathrm{r}^{2}: 0.941\right)$ y un alto índice de correlación (r: 0.970); asimismo, la Sig. (p-valor) es menor a 0.05 (grado de significancia), por lo cual se rechaza la hipótesis nula y se afirma que existe influencia directa y significativa de una dimensión sobre la otra.

Contraste de la hipótesis específica 3

Formulación de la hipótesis nula $\left(H_{0}\right)$

$\mathrm{H}_{0}$ : La consecución de mano de obra calificada del sector construcción no ha incidido notablemente en el producto bruto interno de Moquegua, desde el 2013 al 2017.

$\mathrm{H}_{\mathrm{i}}$ : $\quad$ La consecución de mano de obra calificada del sector construcción ha incidido notablemente en el producto bruto interno de Moquegua, desde el 2013 al 2017.

Nivel de significancia $\propto=0.05$

Pruebas de normalidad

Tabla 16. Pruebas de normalidad para el contraste de la hipótesis especifica 3

\begin{tabular}{lccrrrr}
\hline & \multicolumn{2}{c}{ Kolmogorov-Smirnov } & \multicolumn{3}{c}{ Shapiro-Wilk } \\
& Estadístico & gl & Sig. & Estadístico & gl & Sig. \\
\hline Mano_obra & 0.190 & 20 & 0.057 & 0.878 & 20 & 0.016 \\
PBI & 0.197 & 20 & 0.040 & 0.818 & 20 & 0.002 \\
\hline
\end{tabular}

Fuente: datos procesamiento en Eviews.

Tabla 17. Regresión lineal para el contraste de la hipótesis especifica 3

\begin{tabular}{crrrr}
\hline Modelo & R & R cuadrado & $\begin{array}{r}\text { R cuadrado } \\
\text { corregida }\end{array}$ & $\begin{array}{r}\text { Error típico de la } \\
\text { estimación }\end{array}$ \\
\hline 1 & 0.983 & 0.965 & 0.964 & 79529.660 \\
\hline
\end{tabular}

Fuente: datos procesamiento en Eviews.

Tabla 18. ANOVA para el contraste de la hipótesis especifica 3

\begin{tabular}{crrrrr}
\hline Modelo & Suma de cuadrados & gl & Media cuadrática & F & Sig. \\
\hline Regresión & $3.181 \mathrm{E}+12$ & 1 & $3.181 \mathrm{E}+12$ & 502.963 & 0.000 \\
Residual & $1.138 \mathrm{E}+12$ & 18 & 6324966802 & & \\
Total & $3.295 \mathrm{E}+12$ & 19 & & & \\
\hline
\end{tabular}

Fuente: datos procesamiento en Eviews.

Tabla 19. Coeficientes para el contraste de la hipótesis especifica 3

\begin{tabular}{|c|c|c|c|c|c|}
\hline \multirow{2}{*}{ Modelo } & \multicolumn{2}{|c|}{ Coeficientes no estandarizados } & \multirow{2}{*}{$\begin{array}{r}\begin{array}{r}\text { Coeficientes } \\
\text { estandarizados }\end{array} \\
\text { Beta }\end{array}$} & \multirow{2}{*}{$\mathbf{t}$} & \multirow{2}{*}{ Sig. } \\
\hline & Beta & Error típico & & & \\
\hline (Constante) & 2783678.698 & 278108.492 & & 10.009 & 0.000 \\
\hline Inversión & 42284.842 & 1865.457 & 0.983 & 22.427 & 0.000 \\
\hline
\end{tabular}

Fuente: datos procesamiento en Eviews.

Se observa un alto coeficiente de determinación ( $\left.\mathrm{r}^{2}: 0.965\right)$ y un alto índice de correlación ( $\mathrm{r}$ : 0.983); asimismo, la Sig. (p-valor) es menor a 0.05 (grado de significancia), por lo cual se rechaza la hipótesis nula y se afirma que existe influencia directa y significativa de una dimensión sobre la otra. 


\section{DISCUSIÓN}

Los resultados muestran un incremento de la actividad del sector construcción en un $13.1 \%$ en el 2017, a comparación del 2013, y un $6.1 \%$ a comparación del 2016. Este aumento notable tiende a ser positivo hacia años posteriores, aun cuando sucediese una desaceleración, seguirá siendo importante para el desarrollo económico de Moquegua.

Se han considerado tres elementos que son importantes para determinar la inversión privada en construcción: el capital, las licencias y la mano de obra calificada; los cuales, en caso faltase alguno de estos elementos, las edificaciones no serían posibles y conllevaría a un retraso en la ciudad y la región.

En el primer caso, la inversión de capital ha presentado un decrecimiento del $1.9 \%$ en el consumo interno de cemento, un aumento del $9.9 \%$ en la adquisición de barras de construcción y un $22.6 \%$ en la venta de asfalto, los cuales constituyen la materia prima más importante en las obras de edificación y servicio público.

Respecto a las licencias de construcción tramitadas, se ha denotado un incremento del $64.6 \%$ en el 2017, a comparación del 2013; además de ser constante, lo cual refleja un crecimiento, de manera formal, de las edificaciones y la tercerización de obras públicas.

En caso de la mano de obra calificada, la cual fue capacitada por el Servicio Nacional de Capacitación para la Industria de la Construcción - SENCICO, ha presentado un aumento de egresados, dispuestos a prestar sus servicios en el rubro de la construcción, en un 20.7 \%. Este elemento fortalece la dinámica del sector construcción porque cubrirá las plazas requeridas para realizar las edificaciones particulares y obras públicas; en cambio si la inversión privada se detiene o desacelera; entonces, podría afectar la situación económica y laboral de la mano de obra calificada.

Entonces, estos tres indicadores que son importantes para el crecimiento del sector construcción están relacionados con el producto bruto interno, demostrados en el análisis estadístico, a través de la prueba regresión lineal, en donde se rechazó la hipótesis nula que negaba la incidencia de la inversión privada en el PBI de Moquegua y se obtuvo un coeficiente de determinación de 0.962 y de correlación de 0.981 (tablas 7, 8 y 9), los cuales indican una influencia significativa y positiva.

En concordancia con Mero, Herrera y Herrera (2018), las actividades de inversión sostenible en la construcción participan notablemente en el PBI, hay que considerar que su estudio se realizó a nivel nacional (Ecuador), en donde una provincia puede contribuir más que otra, una realidad similar a la peruana, en donde la construcción está más fuertemente ligada a las ciudades urbanas más pobladas y en actividades extractivas e industriales, que suelen ubicarse en la costa y sierra del país, mas no en la selva.

Entre los hallazgos de Pineda (2013), se ubica la capacitación de los empleados, por lo cual no se logró determinar la productividad de las empresas del sector construcción. Esta situación adversa refleja la importancia de la mano de obra calificada para la realización de este tipo de actividad económica, aunque suele costearse más, constituye una garantía para contribuir al crecimiento económico de la región.

Respecto a Frey y Vela (2014), resaltan la necesidad de reducir la brecha de infraestructura y el déficit de viviendas en el país, lo cual hace que el mercado se vea atractivo para la inversión privada en construcción que incentivará un consumo interno mayor de materias primas como cemento, barras y asfalto, con la finalidad de garantizar el crecimiento socioeconómico y mejorar la calidad de vida.

Se concuerda con Vargas, Castro y Bautista (2011), en que el sector construcción es un elemento principal del crecimiento económico del país, por ello, debe crecer en paralelo con los demás sectores, como minería, pesquería, obras públicas, industria y vivienda, porque les provee de infraestructura para la realización de sus actividades económicas. 


\section{REFERENCIAS}

Campos, E. F., y Carcelén, C. F. (2003). Diseño e implementación de una estructura organizativa y aplicación de las técnicas de personal en una empresa constructora y de alquiler de maquinaria [Tesis]. Universidad de Piura.

Cerda, R., y Larraín, F. (2005). Inversión privada e impuestos corporativos: evidencia para Chile. Cuadernos de Economia, 42( 126 ). D is po n i b 1 e e n https://scielo.conicyt.cl/scielo.php?script=sci_arttext\&pid=S0717-68212005012600003

Flores, C. (15 de octubre de 2018). INEI: El sector construcción cae 0,09 \% en agosto. Diario Correo. Disponible en https://diariocorreo.pe/economia/inei-el-sector-construccion-cae-009-enagosto-847827/

Frey, J. C., y Vela, J. P. (2014). Relevancia del proceso de abastecimiento en empresas grandes del sector construcción: Estudio de caso comparado entre empresas familiares peruanas [Tesis]. Pontificia Universidad Católica del Perú.

González, J. (2016). ¿Qué sucede con la inversión privada? El Comercio. Disponible en https://elcomercio.pe/opinion/colaboradores/sucede-inversion-privada-jorge-gonzalez-i223789

Jiménez, F. (2010). La economía peruana del último medio siglo: ensayos de interpretación. Lima: CISEPA-PUCP.

Kogan, J., y Bondorevsky, D. (2016). La infraestructura en el desarrollo de América Latina. Economía y $\begin{array}{llll}D \text { es ar rollo, } & 156(1) . & \text { D is pon i b l e } & \text { e n }\end{array}$ http://scielo.sld.cu/scielo.php?script=sci_arttext\&pid=S0252-85842016000100012

Lezama, C. (24 de marzo de 2018). Plan de desarrollo de Moquegua denota liderazgo. El Peruano, Especial, p.6.

Mendoza, J. (2016). La inversión privada. Diario Gestión. Disponible en https://gestion.pe/blog/economia-aplicada/2016/07/la-inversion-privada-por-juanmendoza.html?ref=gesr

Mero, I. C., Herrera, M. V., y Herrera, J. E. (2018). Influencia de la sostenibilidad en el sector de la construcción en Ecuador sobre el producto interno bruto PIB período 2010-2016. Observatorio de la Economia Latinoamericana. D i s po n i b 1 e e n https://www.eumed.net/rev/oel/2018/07/construccion-ecuador-pib.html

Pineda, M. A. (2013). Análisis de la productividady sus determinantes en el sector de la construcción del Ecuador en base al censo económico (Tesis). Facultad Latinoamericana de Ciencias Sociales, sede Ecuador.

Umaña, Y. (2003). El sector de la construcción: Un sector líder. Superfinanciera. Disponible en https://www.superfinanciera.gov.co/descargas/institucional/pubFile1031827/yolimaumana.p df

Vargas, A., Castro, V., y Bautista, E. (2011). Importancia del crecimiento del sector construcción en la economía y sociedad peruana. Gestión en el Tercer Mundo, 14(28), 25-32.

Verona, M. C., y Hernández, M. (2010). Decisiones de inversión y financiación en empresas del sector turístico. In novar, 22(43). $\quad$ D i s p o n i b l e e n http://www.scielo.org.co/scielo.php?script=sci_arttext\&pid=S0121-50512012000100016

Vyas, S., Ahmed, S., y Parashar, A. (2014). Bureau and Energy Efficiency, and Green Buildings. International Journal of Research, 1, 23-32. 